\title{
Bacterial Surface-Displayed Gll.4 Human Norovirus Capsid Proteins Bound to HBGA-Like Molecules in Romaine Lettuce
}

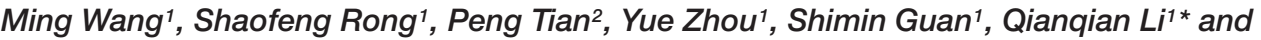 \\ Dapeng Wang ${ }^{3 *}$
}

${ }^{1}$ Department of Bioengineering, Shanghai Institute of Technology, Shanghai, China, ${ }^{2}$ Produce Safety and Microbiology Research Unit, Western Regional Research Center, Agricultural Research Service - United States Department of Agriculture, Albany, CA, USA, ${ }^{3}$ MOST-USDA Joint Research Center for Food Safety, School of Agriculture and Biology, Shanghai Jiao Tong University, Shanghai, China

OPEN ACCESS

Edited by:

David Rodriguez-Lazaro,

University of Burgos, Spain

Reviewed by:

Gloria Sánchez Moragas, Instituto de Agroquímica y Tecnología

de Alimentos - Consejo Superior de Investigaciones Cientificas, Spain

Jacques Le Pendu, Institut National de la Santé et de la Recherche Médicale, France

*Correspondence:

Qianqian L

qqli@sit.edu.cn

Dapeng Wang

norovirus@163.com

Specialty section: This article was submitted to

Food Microbiology,

a section of the journa

Frontiers in Microbiology

Received: 10 January 2017 Accepted: 06 February 2017 Published: 20 February 2017

Citation:

Wang M, Rong S, Tian P, Zhou Y, Guan S, Li Q and Wang D (2017) Bacterial Surface-Displayed Gll.4 Human Norovirus Capsid Proteins Bound to HBGA-Like Molecules in Romaine Lettuce.

Front. Microbiol. 8:251. doi: 10.3389/fmicb.2017.00251
Human Noroviruses (HuNoVs) are the main cause of non-bacterial gastroenteritis. Contaminated produce is a main vehicle for dissemination of HuNoVs. In this study, we used an ice nucleation protein mediated surface display system to present the protruding domain of Gll.4 HuNoV capsid protein on bacterial surface and used it as a new strategy to explore interaction between HuNoV protein and receptor candidates from romaine lettuce. The surface-displayed HuNoV proteins were confirmed on the surface of the transformed bacteria by an immunofluorescence assay. The distribution patterns of the surface-displayed HuNoV proteins in romaine lettuce were identified through a confocal immunofluorescence assay. The surface-displayed HuNoV proteins could be found in the stomata, and the surfaces of vein and leaf of romaine lettuce. The surface-displayed HuNoV proteins could be captured by an ELISA assay utilizing extract from leaf (LE) or vein (VE). The binding of the surface-displayed HuNoV proteins to LE or VE could be competitively blocked by histo-blood group antigens from human saliva. In addition, the binding of the surface-displayed HuNoV proteins to LE or VE could also be attenuated by heat denaturation of lettuce proteins, and abolished by oxidation of lettuce carbohydrates. The results indicated that histo-blood group antigen-like molecules in LE or VE were involved in the binding of the surface-displayed HuNoV proteins to romaine lettuce. All data demonstrated that the surface-displayed HuNoV proteins could be utilized in a new and simple system for investigation of the interaction between the HuNoVs and their candidate ligands.

Keywords: norovirus, cell surface display, capsid protein, protruding domain, HBGAs, romaine lettuce, GIl.4

\section{INTRODUCTION}

Norovirus (NoV) is a single-stranded RNA virus of the family Caliciviridae. Based on the sequence of the major capsid protein (VP1), noroviruses have been categorized into seven genogroups (GI though GVII) (Vinjé, 2015). The GI, GII, and GIV genogroups are capable of infecting humans, and comprise the human noroviruses (HuNoVs). GII strains are more common, and are the main cause of human non-bacterial gastroenteritis worldwide (Hoa Tran et al., 2013). 
Infection by HuNoVs could be associated with the consumption of fresh produce, such as romaine lettuce (Ethelberg et al., 2010), onion (Dicaprio et al., 2015), strawberries (Made et al., 2013), and raspberries (Sarvikivi et al., 2012). Produce could be contaminated before harvest by irrigation water (Mok et al., 2014), manure or bio-solids (Wei et al., 2010). Harvest and post-harvest cross-contamination of produce could result from human contact, including handling, chopping/slicing and mixing (Grove et al., 2015). In addition, HuNoVs are stable in the environment and remain infectious in fresh produce surface for a long time (Verhaelen et al., 2012). Therefore, contaminated produce becomes one of the important vehicles for the transmitting of HuNoVs.

Until recent reports of successful replication of HuNoVs in human B cells (Jones et al., 2015) and stem cell-derived human enteroids (Ettayebi et al., 2016), the lack of an in vitro cultivation system had been a major barrier to the study of HuNoVs. In lieu of HuNoVs, viral-like particles (VLPs) and surrogate viruses including feline Calicivirus (FCV), murine norovirus (MNV), Tulane virus (TV) have been used (Jiang et al., 1992; Bozkurt et al., 2013; Xu et al., 2015). Limited literatures were available on interactions between HuNoVs and ligands from produce, and results were not consistent. GII.4 HuNoV, TV, and MNV were found to internalize into lettuce via roots, and disseminate into shoots and leaves (Dicaprio et al., 2012). Wei et al reported that MNV were found on leaf surfaces, in stomata, and at cut edges of romaine lettuce (Wei et al., 2010). Wang et al reported that porcine sapovirus could attach to romaine lettuce and remain infectious for a week after storage at refrigeration temperatures (Wang et al., 2012). Gandhi et al reported that GI.1 VLPs could be found in clusters along romaine lettuce veins. The binding of GI VLPs to romaine extract (RE) could not be competitively inhibited by porcine gastric mucin (PGM), suggesting that molecules involved in the binding of GI NoV to romaine lettuce might not be related to PGM-like carbohydrates (Gandhi et al., 2010). Esseili et al. (2012) reported that GII.4 VLPs bound to carbohydrates of romaine lettuce leaves. They demonstrated that attachment of GII.4 VLPs to young leaves was primarily associated with proteins whereas for older leaves it was primarily through carbohydrates. However, the binding of VLPs to romaine lettuce leaf cell wall materials could only be partially inhibited by PGM, carbohydrate-binding lectins, and oxidation of carbohydrate. Gao et al. (2016) reported that binding of GII.4 VLPs to romaine lettuce was enhanced after digesting lettuce leaves with cell-wall-degrading enzymes. They further demonstrated that HBGA-like molecules exist within lettuce tissue, and GII.4 VLPs could bind the exposed fucose moiety of HBGAs.

Most studies of the interaction between HuNoVs and receptor candidates on/in romaine lettuce utilized recombinant HuNoV VLPs expressed from eukaryotic expression systems, or P-particles expressed from prokaryotic systems (Tan and Jiang, 2005). It has been well documented that there was no difference between eukaryote-expressed recombinant proteins (VLP) and prokaryote-expressed proteins ( $\mathrm{P}$ particles) in its biological functions such as HBGA binding and immunogenicity (Tan et al., 2008, 2011; Tamminen et al., 2012). However, there are some technical issues on making them. Making recombinant eukaryotic system was complicated and timeconsuming. Expression in Escherichia coli had downstream processing issues due to the presence of inclusion bodies which need to be disrupted to form functional virions by complicated purification steps. The purpose of the study was to develop a new strategy to present noroviral proteins on the surface of the transformed E. coli to avoid complicated purification steps and use this new system to identify candidate receptors for $\mathrm{HuNoV}$ binding in lettuce.

\section{MATERIALS AND METHODS}

\section{Plasmid DNA and Bacteria Transformation}

Escherichia coli BL21 (Thermo Fisher, Shanghai, China) was used as competent cell for recombinant plasmid transformation and expression of the target protein. pET28a-inaQn-P (GII.4) and pET28a-P (GII.4) were constructed as previously described (Niu et al., 2015), pET28a-inaQn was constructed by inserting inaQn into Nco I/BamH I digested pET28a and used as a control. All the recombinant plasmids were used to transform bacteria E. coli BL21. Figures 1A,B presented for the plasmid DNA map and a schematic figure for displayed $\mathrm{P}$ protein on the surface of pET28a-inaQn-P(GII.4) transformed bacteria [INP-P (GII.4) BL21].

\section{Culture and Expression of SD-GII.4P Fusion Protein in E. coli BL21}

Recombinant E. coli BL21 harboring inaQn and $P$ protein fusion gene [named INP-P (GII.4) BL21], $P$ protein gene [named P (GII.4) BL21] and inaQn gene (named INP BL21) were cultured as previously reported (Niu et al., 2015). Cells were cultured in $5.0 \mathrm{ml}$ of LB medium containing $100 \mu \mathrm{g} / \mathrm{ml}$ kanamycin with shaking $(220 \mathrm{rpm})$ at $37^{\circ} \mathrm{C}$, overnight. The cells $(50.0 \mu \mathrm{l})$ were subcultured in $5.0 \mathrm{ml}$ fresh $\mathrm{LB}$ medium with $60 \mu \mathrm{g} / \mathrm{ml}$ kanamycin. When the $\mathrm{OD}_{600}$ reached 0.5 , isopropyl$\beta$-D-thiogalactopyrano-side (IPTG) was added to reach a final concentration of $0.4 \mathrm{mmol} / \mathrm{l}$. The cells were incubated at $25^{\circ} \mathrm{C}$ for $12 \mathrm{~h}$, then washed and diluted to $\mathrm{OD}_{600}=1.0$ with sterile PBS, and then kept at $4^{\circ} \mathrm{C}$ for further use.

\section{Identification of Surface Displayed P Proteins by Immunofluorescence Assay}

The localization of surface-displayed P protein (SD-GII.4P) was examined using immunofluorescence microscopy (Olympus, Japan) as previously described (Li et al., 2009). Anti-VP1 (GII.4) polyclonal antisera from immunized Balb/C mice (1:5000 in PBS) were used as the primary antibody (Niu et al., 2015), and 1:100diluted FITC-conjugated goat anti-mouse IgG (Thermo Fisher, USA) was used as the secondary antibody. The anti-VP1 (GII.4) polyclonal antisera were pre-absorbed with E. coli BL21 extract to reduce potential non-specific. Three sets of negative controls were used. P (GII.4) BL21, INP BL21, and E. coli BL21 were 

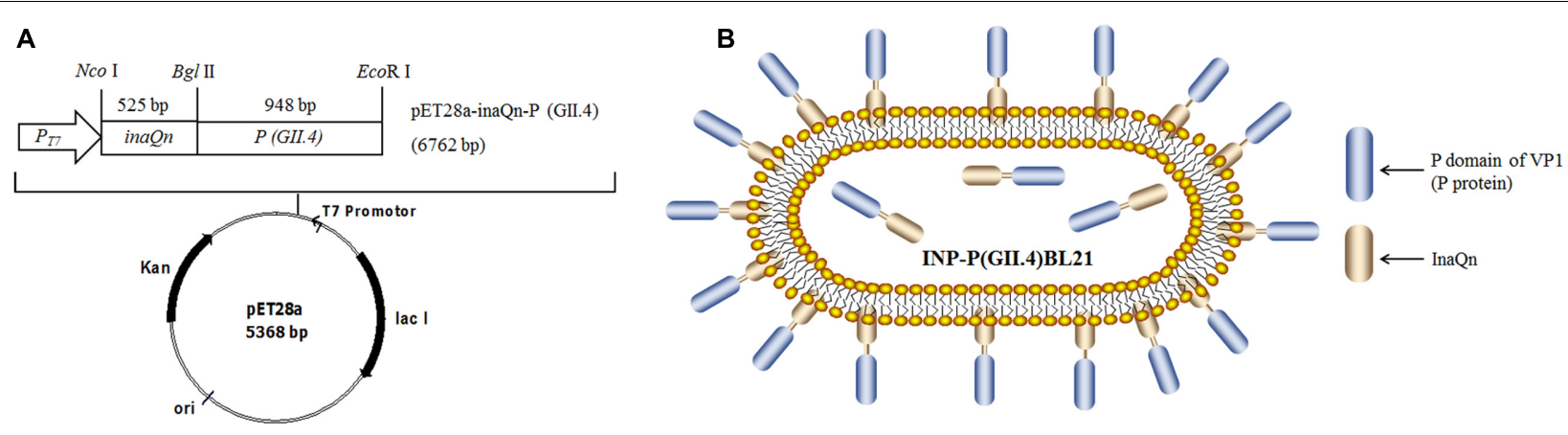

FIGURE 1 | (A) Plasmid DNA map; (B) Schematic figure for SD-Gll.4P displayed on the surface of pET28a-inaQn-P (GIl.4) transformed bacteria [INP-P (GIl.4) BL21]. inaQn: N-terminal of ice nucleation protein encoding gene; P(Gll.4): P domain of Gll.4 HuNoVs capsid protein encoding gene; $P_{T 7}$ : T7 promoter; Nco I, Bgl II and EcoR I: restriction enzymes; Kan: kanamycin - resistant gene; ori: pBR322 origin; lac I: overexpressed lac repressor.

used to test non-specific binding of polyclonal antisera. INP-P (GII.4) BL21 without primary antibody (with PBS only) was used as additional negative control for non-specific binding of FITC-conjugated secondary antibody.

\section{Preparation of Romaine Lettuce}

Romaine lettuce was collected randomly from a local grocery store and stored at $4^{\circ} \mathrm{C}$. The leaves were washed three times with sterile distilled water. For microscopy, approximately $2 \mathrm{~cm}^{2}$ of the strips were cut from the ends of leaves using a sterile blade. For ELISA, the romaine lettuce was made into leaf extract (LE) and vein extract (VE), collectively referred to as RE. LE was prepared from the top half of a romaine leaf $(15 \mathrm{~g})$, while VE was prepared from the excised main veins. The same volume of PBS (wt/vol) was added to each vegetable matter sample, and blended for $5 \mathrm{~min}$ at $4^{\circ} \mathrm{C}$. The liquid phase was transferred to a sterile microcentrifuge tube and centrifuged at $10,000 \times g$ for $10 \mathrm{~min}$ at $4^{\circ} \mathrm{C}$. The supernatant was transferred to a fresh sterile tube and kept at $4^{\circ} \mathrm{C}$ for further use.

\section{Preparation of Boiled Saliva}

Human saliva was collected from A, B, O blood type volunteers and treated according to the previous report, with minor modification (Wang et al., 2014). The secretor status of individual saliva was indirectly determined by an ELISA assay for its ability to bind purified bacteria-expressed GI and GII VP1 and $\mathrm{P}$ domains (Niu et al., 2015) and directly determined with corresponding monoclonal antibodies. Although $\mathrm{HuNoV}$ binding ability was confirmed in all saliva tested, three saliva samples from the same blood type were mixed and used for competitively assays. The study was approved by the Institutional Bio-safety Committees (IBC) of College of Agriculture and Biology, Shanghai Jiao Tong University, and written informed consent was obtained from the volunteers. Briefly, each type of saliva was collected from at least three volunteers and mixed. Then, each saliva sample was boiled for $5 \mathrm{~min}$, and then followed with centrifugation at $10,000 \times g$ for $5 \mathrm{~min}$ at $4^{\circ} \mathrm{C}$. The individual supernatant of saliva from each blood group was mixed, aliquoted and stored at $-20^{\circ} \mathrm{C}$ for further use. Saliva from non-secretors was used as negative control.

\section{Identification of SD-GII.4P Binding to Romaine Lettuce by Confocal Immunofluorescence Assay}

Strips of romaine lettuce on glass slides were blocked with 1\% bovine serum albumin (BSA, Yeasen, Shanghai, China) in PBS at $4^{\circ} \mathrm{C}$, overnight, and then soaked in sterile PBS for $5 \mathrm{~min}$. The strips were moved into a sterile plate containing INP-P (GII.4) BL21 or E. coli BL21 (negative control), and incubated at room temperature for $1 \mathrm{~h}$. The strips were then washed with PBS solution and put on a slide. Then, $70 \mu \mathrm{l}$ of primary antibody (Niu et al., 2015) at a dilution of 1:2,000 in PBS was added onto the surface of strips and incubated at room temperature for $1 \mathrm{~h}$. After incubation, strips were washed three times with PBS. A volume of $50 \mu \mathrm{l}$ FITC-conjugated goat anti-mouse IgG $(\mathrm{H}+\mathrm{L})$ (Yeasen, Shanghai, China) at a dilution of 1:100 in PBS was added onto the strips, and incubated at room temperature for $1 \mathrm{~h}$. After washing 3 times with PBS, $30 \mu \mathrm{l}$ PBS was applied to the surface of the romaine, covered with a coverslip, and observed under a confocal laser scanning microscope (Leica Microsystems, Wetzlar, Germany). The images were magnified at $700 \times$.

\section{Romaine Lettuce Extract-Binding or PGM-Binding Based ELISA}

The RE-binding enzyme-linked immunosorbent assay (ELISA) was performed as previously reported (Gandhi et al., 2010), with minor modification. Briefly, LE or VE was diluted in PBS (1:5, $\mathrm{pH}=7.4$ ) (Gandhi et al., 2010), and PGM (Sigma, MI, USA) was dissolved in carbonate buffer solution $(\mathrm{pH}=9.6)$ to $1.0 \mathrm{mg} / \mathrm{ml}$ (Wang et al., 2014). Then, LE, VE, or PGM was added into and allowed to coat 96-well microtiter plates (100 $\mu$ l per well) at $4^{\circ} \mathrm{C}$ overnight. Plates were washed three times with PBS, and blocked with $1 \% \mathrm{BSA}$ at $37^{\circ} \mathrm{C}$ for $2 \mathrm{~h}$. Plates were washed three times with PBS containing 0.1\% Tween-20 (PBS-T). The INP-P (GII.4) BL21, E. coli BL21, P (GII.4) BL21, and INP BL21 samples $(100 \mu \mathrm{l})$ were added to each well, respectively, and incubated at 
$37^{\circ} \mathrm{C}$ for $1 \mathrm{~h}$. After washing three times with PBS-T, $100 \mu \mathrm{l}$ of primary antibodies (1:10,000 in PBS) was added to each well, and incubated at $37^{\circ} \mathrm{C}$ for $1 \mathrm{~h}$. After washing three times with PBS-T, $100 \mu \mathrm{l}$ of peroxidase-conjugated goat anti-mouse IgG $(\mathrm{H}+\mathrm{L})$ (Yeasen, Shanghai, China) at a dilution of 1:5,000 in PBS was added to each well, and incubated at $37^{\circ} \mathrm{C}$ for $1 \mathrm{~h}$. After washing five times with PBS-T, $100 \mu \mathrm{l}$ 3,3',5,5'-tetramethylbenzidine (Yeasen, Shanghai, China) was added to each well. Plates were kept in the dark for $5 \mathrm{~min}$, and then $50 \mu \mathrm{l}$ of $2.0 \mathrm{~mol} / \mathrm{l} \mathrm{H}_{2} \mathrm{SO}_{4}$ was added to stop the reaction. $\mathrm{OD}_{450}$ value was measured using a Microplate Reader (Sanjose, Shanghai, China). In addition, to determine if INP could bind to RE, INP BL21 was added to RE coated wells, followed by antibodies against INP (prepared as described in Li et al., 2009) and peroxidase-conjugated secondary antibody.

Romaine extract-coated or PGM-coated wells with PBS were used as the blank control. The bacteria E. coli BL21 was used as a negative control $(\mathrm{N})$. Samples were considered as positive when the positive to genitive $(\mathrm{P} / \mathrm{N})$ ratio was greater than 2.0 .

\section{Competitive Inhibition ELISA Assay}

Boiled mixed saliva was diluted five times with PBS. The INP-P (GII.4) BL21 $(100 \mu \mathrm{l})$ cells were incubated with the diluted boiled saliva $(100 \mu \mathrm{l})$ or $1.0 \mathrm{mg} / \mathrm{ml}$ PGM $(100 \mu \mathrm{l})$ solution at $37^{\circ} \mathrm{C}$ for $30 \mathrm{~min}$. After centrifugation at $10,000 \times g$ for $2 \mathrm{~min}$ at $4^{\circ} \mathrm{C}$, the precipitated INP-P (GII.4) BL21 was re-suspended in PBS ( $\mathrm{pH}=7.4$ ). The INP-P (GII.4) BL21 incubated with PBS was used as untreated control. E. coli BL21 (negative control) was treated with the same process. After blocking, the INP-P (GII.4) BL21 was detected by RE-binding based ELISA as described in the Section "Romaine Lettuce Extract-Binding or PGM-Binding Based ELISA". To calculate percentage inhibition, the $\mathrm{P} / \mathrm{N}$ ratio of untreated samples was equal to $0 \%$ inhibition and $\mathrm{P} / \mathrm{N}$ ratio of 2.0 was equal to $100 \%$ inhibition.

\section{Protein denaturation or carbohydrate oxidation of LE and VE}

Porcine gastric mucin was used as a positive control for protein denaturation and carbohydrate oxidation (Tian et al., 2005, 2007). LE or VE was boiled for $5 \mathrm{~min}$, and centrifuged at $10,000 \times g$ for $10 \mathrm{~min}$ at $4^{\circ} \mathrm{C}$. The supernatant was diluted five times with carbonate buffer solution $(\mathrm{pH}=9.6)$, and was used to coat the wells as described in Section "Romaine Lettuce Extract-Binding or PGM-Binding Based ELISA". To determine whether carbohydrates were involved in INP-P (GII.4) BL21 binding, $100 \mu l 4.0 \mathrm{mg} / \mathrm{ml} \mathrm{NaIO}_{4}$ (Adamas Reagent Co., Ltd., Shanghai, China) was added to wells coated with LE or VE (Esseili et al., 2012). The plates were incubated at $37^{\circ} \mathrm{C}$ for $30 \mathrm{~min}$ prior to the addition of the INP-P (GII.4) BL21. PGM, LE, or VE without protein denaturation or oxidation was used as untreated controls. After treatment, the binding capability and percentage of inhibition were evaluated as described in Sections "Romaine Lettuce Extract-Binding or PGM-Binding Based ELISA" and "Competitive Inhibition ELISA Assay”.

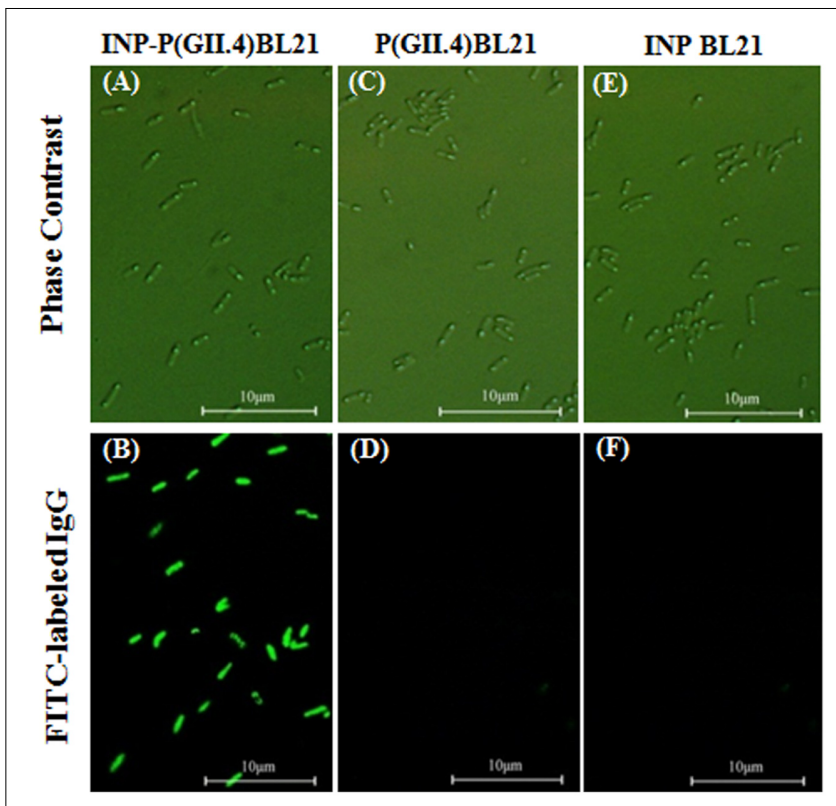

FIGURE 2 | Surface localization of SD-GII.4P. INP-P (GIl.4) BL21, P (GIl.4) $B L 21$, and INP BL21 were observed under phase contract microscopy (A,C,E), and fluorescence microscopy (B,D,F).

\section{The pH Effects on the Interaction between INP-P (GII.4) BL21 and Romaine Lettuce}

The INP-P (GII.4) BL21 and E. coli BL21 (negative control) was diluted across a range of $\mathrm{PBS}$ of different $\mathrm{pHs}$, and adjusted to the same $\mathrm{OD}_{600}$ value. The INP-P (GII.4) BL21 was detected by REbinding-based ELISA as described in Section "Romaine Lettuce Extract-Binding or PGM-Binding Based ELISA". $\mathrm{P} / \mathrm{N}$ ratio at $\mathrm{pH}$ 6.4 was equal to $100 \%$ to calculate the percentage of binding.

\section{Statistics}

Each experiment $(\mathrm{N})$ was performed in triplicate $(n=3)$ and repeated at least three times $(N>3)$. The means and standard deviations from independent experiments were presented in all figures. One-way analysis of variance (ANOVA) was utilized for data comparison. Differences in means were considered significant when the $p<0.05$.

\section{RESULTS}

\section{SD-GII.4P was Presented at the Surface of the Transformed E. coli BL21}

To verify the surface localization of SD-GII.4P on INP-P (GII.4) BL21 cells, immunofluorescence assay was performed (Figure 2). SD-GII.4P fusion proteins were clearly visualized as fluorescent spots on the transformed cell surface (Figures 2A,B). No FITC fluorescence signals were observed in P (GII.4) BL21 (Figures 2C,D), INP BL21 samples (Figures 2E,F) and E. coli BL21 (data not shown). 


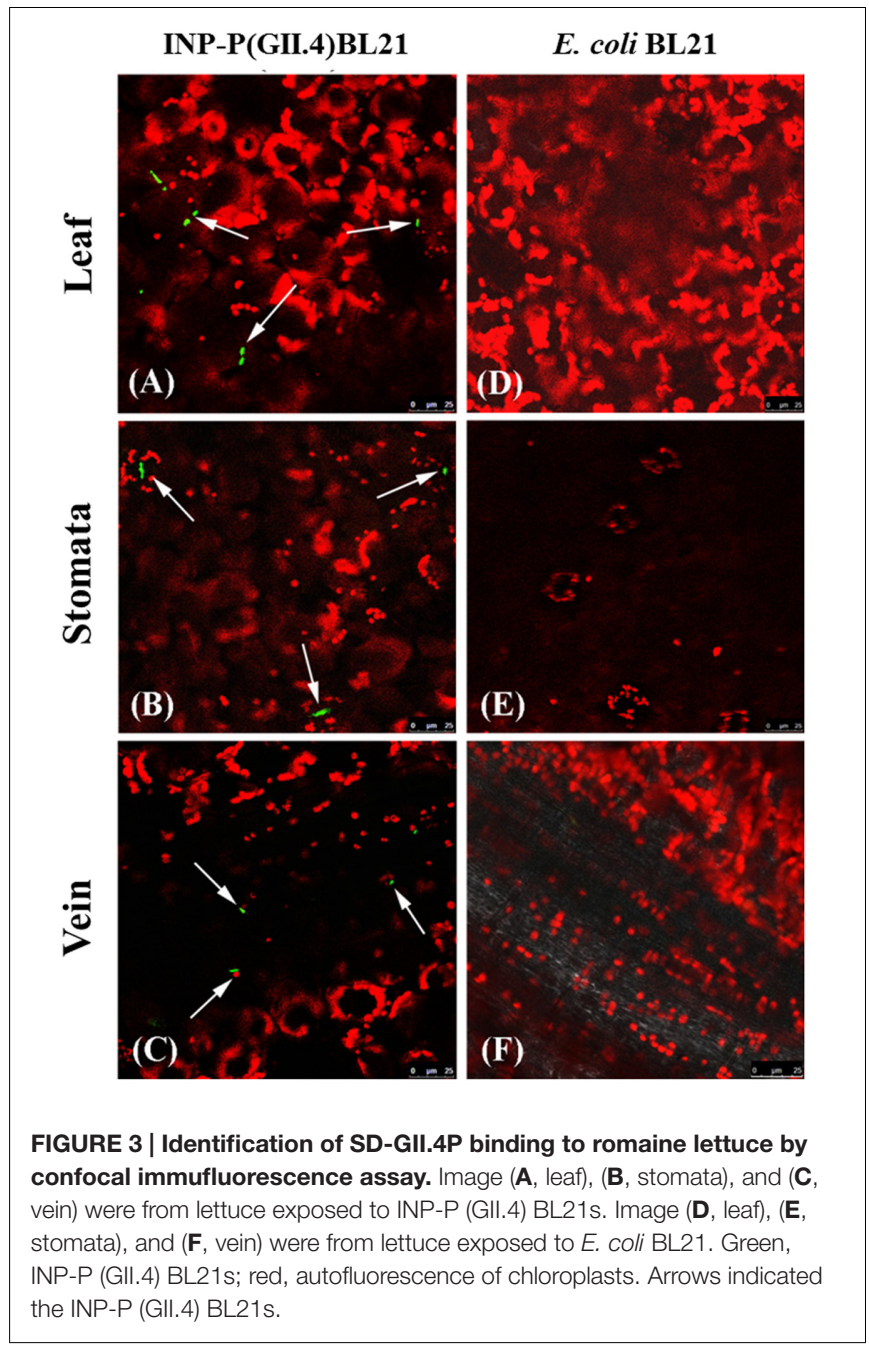

\section{Distribution of INP-P (GII.4) BL21 on Romaine Lettuce Surface}

The INP-P (GII.4) BL21 bacteria were observed directly by Confocal Laser Scanning Microscope using FITC-labeled antibodies. After being washed, the INP-P (GII.4) BL21 bacteria were found to be distributed on the leaf (Figure 3A), stomata (Figure 3B) and vein (Figure 3C) of romaine lettuce. Romaine lettuce with E. coli BL21 bacteria exhibited no green fluorescent signals on its surface (Figures 3D-F). The red signals were autofluorescence of chloroplasts (Figure 3).

\section{INP-P (GII.4) BL21 Bound to LE and VE and the Binding Could Be Competitively Inhibited by HBGAs in Human Saliva}

The $\mathrm{OD}_{450}$ reading of $E$. coli BL21 was used as control $\mathrm{N}$ to calculate $\mathrm{P} / \mathrm{N}$ ratio in $\mathrm{RE}$ binding based ELISA and inhibition assays. The P/N ratio of P (GII.4) BL21 and INP BL21 were all around 1.0 for LE and VE with antibodies against VP1 of GII.4 $\mathrm{HuNoV}$ (Figure 4). There were no significant differences between these two samples and E. coli BL21. The result suggested that

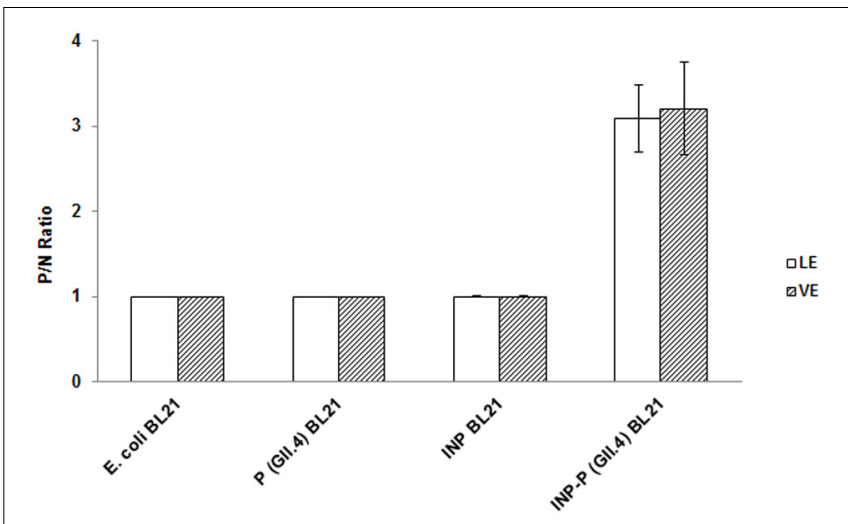

FIGURE 4 | Romaine extract (RE)-based ELISA assays of $E$. coli BL21, P (GII.4) BL21, INP BL21, and INP-P (GII.4) BL21. LE, leaf extract; VE, vein extract. The means and standard deviations from independent experiments were presented. Error bars represent standard deviation.

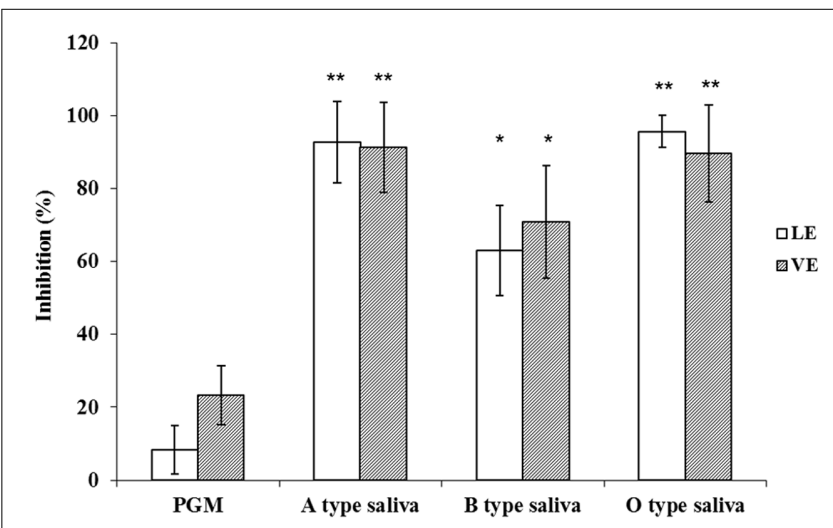

FIGURE 5 | Competitive inhibition ELISA assays. Inhibition ability was shown as percentage. $L E$, leaf extract; $V E$, vein extract; PGM, porcine gastric mucin; A type saliva, B type saliva, and $O$ type saliva indicated A, B, and $O$ blood type saliva, respectively. The means and standard deviations from independent experiments were presented. Error bars represent standard deviation. Statistical differences of $p<0.05$ were indicated by an asterisk and $p<0.01$ were indicated by two asterisks.

BL21 and InaQN could not bind to RE measured by $\mathrm{HuNoV}$ specific antibodies. In addition, with InaQN specific antibodies, we demonstrated that INP BL21 could not bind to RE coated wells as its $\mathrm{P} / \mathrm{N}$ ratio was closed to E. coli BL21 control (data not shown). We further demonstrated that HuNoV P proteins expressed in P (GII.4) BL21 were not be able to bind RE due to its nature of intracellular expression. However, INP-P (GII.4) BL21 could bind to wells coated with LE or VE. The $\mathrm{P} / \mathrm{N}$ ratios ranged from 2.38 to 4.01 for $\mathrm{LE}$ and 2.40 to 4.21 for VE. A slight stronger but not significant binding of INP-P (GII.4) BL21 was observed in VE $(3.21 \pm 0.92)$ than that of LE $(3.09 \pm 0.84)$. The average inhibition rates were $92.68 \pm 11.27 \%$, $95.64 \pm 4.46 \%, 62.98 \pm 12.25 \%$, and $8.31 \pm 6.57 \%$ when INP-P (GII.4) BL21 was pre-incubated with type A, O, B saliva, or PGM (Figure 5) prior to being added to LE-coated wells. Similarly, the average inhibition rates were $91.32 \pm 12.31 \%, 89.55 \pm 13.37 \%$, 


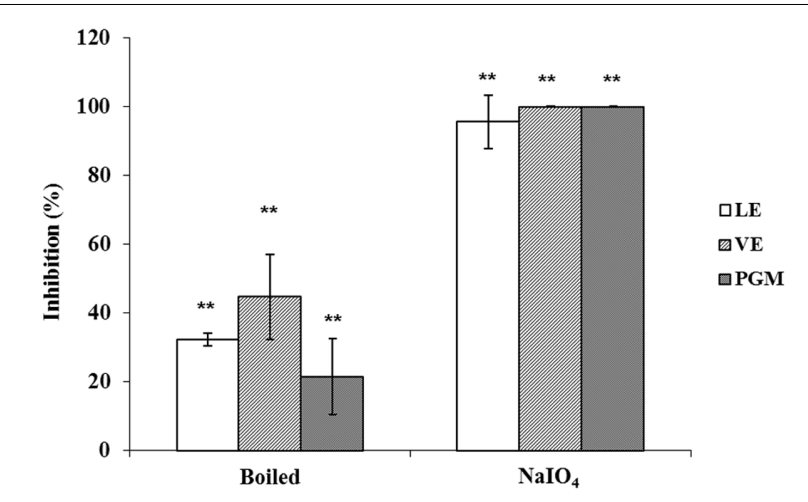

FIGURE 6 | Protein denaturation and carbohydrate oxidation of LE, VE, and PGM. Inhibition ability was shown as percentage. LE, leaf extract; VE, vein extract; PGM, porcine gastric mucin. The means and standard deviations from independent experiments were presented. Error bars represented standard deviation. Statistical differences of $p<0.01$ were indicated by two asterisks.
$70.80 \pm 15.37 \%$, and $23.36 \pm 8.11 \%$ when INP-P (GII.4) BL21 was pre-incubated with type A, O, B saliva, or PGM prior to being added to VE-coated wells (Figure 5). The binding of INP-P (GII.4) BL21 to both LE and VE could be completely inhibited by type-A and -O saliva, and partially inhibited by PGM and type-B saliva. There was no significant difference between the binding of INP-P (GII.4) BL21 to leaf and vein areas of romaine lettuce. In addition, the binding between INP-P (GII.4) BL21 and LE or $\mathrm{VE}$ could not be inhibited by saliva from non-secretors (data not shown).

\section{Characterization of Components in Romaine Lettuce Binding with INP-P (GII.4) BL21}

To determine the nature of the receptor/ligand of romaine lettuce involved in binding INP-P (GII.4) BL21, LE, and VE were either boiled to denature proteins, or oxidized by $\mathrm{NaIO}_{4}$ to remove the functional groups of carbohydrates. It has been reported that HBGAs in PGM were involved in the binding of HuNoV (Tian et al., 2005). Therefore, PGM was used as positive control for the assay. The effects of protein denaturation and carbohydrate oxidation on LE and VE were similar to that of the same to PGM. Wells coated with $\mathrm{NaIO}_{4}$-oxidized LE, VE or PGM resulted in complete inhibition of binding with INP-P (GII.4) BL21 with inhibition rates of $95.49 \pm 7.81 \%, 100 \%$, and $100 \%$, respectively (Figure 6). A significant difference in binding inhibition was observed between $\mathrm{NaIO}_{4}$-treated and untreated reactions with the three groups, but no significant difference was observed between the three groups $(p>0.05)$. Partial inhibition was observed when INP-P (GII.4) BL21 was applied to wells coated with LE, VE or PGM treated with heat to denature proteins (Figure 6). When PGM, LE and VE were heat-treated the inhibition rates were $21.48 \pm 11.09 \%$, $32.23 \pm 1.76 \%$, and $44.66 \pm 12.36 \%$, respectively. There was a significant difference between these three groups treated with or without heat-treatment but no significant difference among

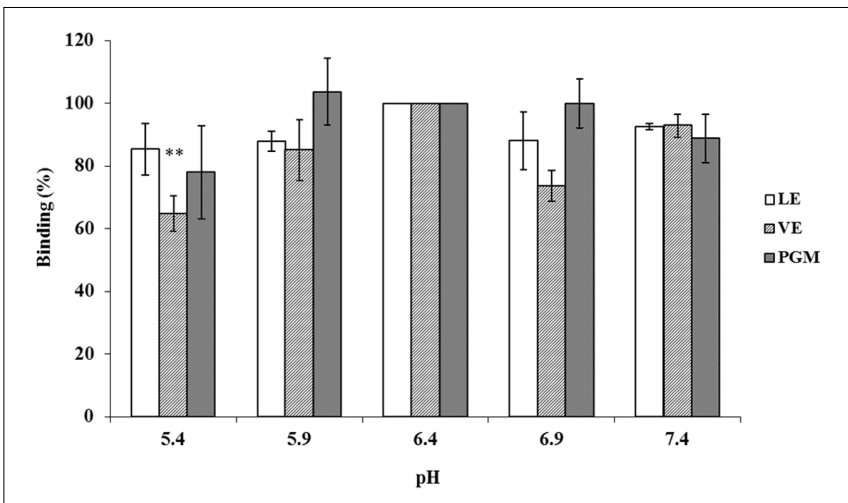

FIGURE 7 | The pH effects on the interaction between INP-P (GII.4) BL21s and romaine lettuce. Binding ability was shown as percentage. LE, leaf extract; VE, vein extract; PGM, porcine gastric mucin. The means and standard deviations from independent experiments were presented. Error bars represented standard deviation. Statistical differences of $p<0.01$ were indicated by two asterisks. these three groups $(p>0.05)$. These results demonstrated that carbohydrates rather than proteins played an important role in $\mathrm{LE}$ and VE for HuNoV binding.

\section{Effect of $\mathrm{pH}$ on the Binding of INP-P (GII.4) BL21 to LE, VE, and PGM}

To determine the effect of $\mathrm{pH}$ on the binding of INP-P (GII.4) BL21 bacteria to romaine lettuce, especially in the vicinity of isoelectric point $(\mathrm{pI})$ of the $\mathrm{P}$ domain protein $(\mathrm{pI}=6.4)$, LE or $\mathrm{VE}$ were treated at conditions below, above, and at $\mathrm{pI}$ of $\mathrm{P}$ protein (Figure 7). PGM was used as a control. Aside from a significant reduction of binding between INP-P (GII.4) BL21 to VE at pH 5.4 $(p<0.004)$, there was no significant difference between binding of INP-P (GII.4) BL21 bacteria to PGM, LE, or VE at various $\mathrm{pHs}$ tested.

\section{DISCUSSION}

Contaminated fresh produce is one of the main causes of $\mathrm{HuNoV}$ infections around the world (Ethelberg et al., 2010). However, the mechanisms of interactions between viruses and fresh produce are poorly understood. As the virus cannot be easily cultured in vitro, it is difficult for many labs to collect enough HuNoVs to perform direct studies. Although VLPs and $\mathrm{P}$ particles were developed as surrogates for $\mathrm{HuNoV}$, the preparation of these surrogates are themselves complicated. Making VLPs from recombinant eukaryotic systems is both complicated and timeconsuming. $\mathrm{P}$ particles could be produced in a prokaryotic system, but are expressed intracellularly and therefore require purification, enzyme digestion, and re-purification steps. In addition, the small sizes of VLPs and $\mathrm{P}$ particles make microscopic observation difficult, and low-speed centrifugation isolation of the viral protein-ligand/receptor complex impossible. In this study, we demonstrated that the $\mathrm{P}$ proteins expressed by P (GII.4) BL21 could not be expressed on the surface of 
bacteria and could not bind to RE extracts (Figures 2 and 4). Therefore, we engineered a novel system to present HuNoV VP1 or P proteins on the surface of transformed bacteria (Niu et al., 2015). The new system uses ice nucleation protein (INP)-target fusion protein to display the target protein on the surface of transformed E. coli BL21. It has been shown that by transforming bacteria with a gene encoding for a fusion target protein with the anchoring motifs of INP, the target protein could be directly displayed on the surface of the bacteria (Cochet and Widehem, 2000; Li et al., 2009, 2012). The advantages of this surface display system are: (1) compared with viruses, bacteria are easy to culture, collect and observe under microscope. (2) Compared with VLPs or $\mathrm{P}$ particles, this surface display system can allow protein products to interact with receptors/ligands directly, requiring neither cell disruption nor protein purification steps. In our previous study, we demonstrated that SD-GII.4VP1 and SDGII.4P could recognize and bind HBGAs (Niu et al., 2015). SD-GII.4P showed a better HBGA-binding ability than that of SD-GII.4VP1 and was selected for use in this study as a model to determine the interactions between the HuNoVs and romaine lettuce.

Surface localization of SD-GII.4P was confirmed by immunofluorescence assay (Figure 2). The SD-GII.4P could be found on the leaf surfaces, stomata and veins of romaine lettuce (Figure 3). The results were consistent with previous reports. Gandhi et al. (2010) reported that GI.1 VLPs could be found in clusters along the veins. Esseili et al. (2012) reported that GII.4 VLPs bound to leaves of romaine lettuce. Finding SD-GII.4P in stomata in this study is interesting as stomata might provide protection to viruses from liquid disinfectants or simple washing. It is common to find bacteria in stomata because of the move ability of bacteria (Xicohtencatl-Cortes et al., 2009; Golberg et al., 2011; Kroupitski et al., 2011). Wei et al reported that MNV could be found in stomata, at the cut edges and on leaf surfaces of romaine lettuce (Wei et al., 2010). DiCaprio et al reported that HuNoV VLPs, TV, and MNV could be found in stomata (Dicaprio et al., 2012). It remains unknown when and how these viruses/VLPs got into stomata, and their entry with or without binding to bacteria. In this study, we reported that SD-GII.4P could get into stomata via bacteria. As some enteric bacteria are capable of expressing HBGAs that bind HuNoVs, it raises the possibility that enteric bacteria might facilitate the entry of HuNoVs into stomata, which confers protection from washing and disinfection processes. This could occur by either HuNoVs binding to bacterial HBGAs prior to the latter's entry into stomata, or binding to bacteria already in stomata.

Vega et al used four different viruses to determine the effect of $\mathrm{pH}$ on viral attachment to lettuce (Vega et al., 2005). The results showed that the pI of viruses was not the governing factor in their attachment to lettuce. Similarly, our results also indicated that the $\mathrm{pI}$ of $\mathrm{P}$ protein was not a major factor in the binding between INP-P (GII.4) BL21 and lettuce (Figure 7). As the bacteria were not stable in a lower or higher $\mathrm{pH}$, only $\mathrm{pH}$ around the $\mathrm{pI}$ of $\mathrm{P}$ domain were tested. Although no charge exists on the surface of $\mathrm{P}$ protein at $\mathrm{pI}$ ( $\mathrm{pH}$ 6.4), INP-P (GII.4) BL21 still exhibits a binding ability to RE, suggesting a specific binding between receptor and ligand. To determine if specific molecules in romaine lettuce were involved in the binding of SD-GII.4P, a competitive binding assay was performed (Figure 5). The binding of SD-GII.4P to LE and VE could be completely inhibited by human saliva containing type-A or -O HBGAs, and could be partially inhibited by human saliva containing type-B HBGAs. The binding ability of LE and VE could also be abolished when their carbohydrates are oxidized by $\mathrm{NaIO}_{4}$ (Figure 6). All these results suggest that type-A and -O HBGAs-like molecules in LE and VE were responsible for $\mathrm{HuNoV} \mathrm{P}$ protein binding. Our results were consistent with reports from other groups. Gao et al reported that monoclonal antibodies against HBGAs could inhibit the interaction between the GII.4 VLPs and lettuce leaf (Gao et al., 2016). Esseili et al. (2012) reported that multiple carbohydrate moieties, mainly $\alpha-D-G a l$, GalNAc, Man/Glc, Fuc, GlcNAc, and sialic acid, were involved in the binding of GII.4 NoV VLPs to leaf cell wall materials of romaine lettuce. Gandhi et al. (2010) reported that GI.I VLPs could bind to romaine lettuce veins and this binding could not be completely inhibited by PGM. Similarly, our results also indicated that PGM could not completely inhibit the binding of SD-GII.4P to LE and VE. It is possible that HBGAs present in human saliva was much higher than that of PGM used in the study.

It was reported that the isolated $\mathrm{P}$ protein could spontaneously dimerize, and stabilized the protein in solution. In addition, the $\mathrm{P}$ dimers could also combine to form $\mathrm{P}$ particles, which were composed of 12 sets of $\mathrm{P}$ dimers. Both the $\mathrm{P}$ dimer and $\mathrm{P}$ particle were capable of binding HBGAs (Tan and Jiang, 2005; Cao et al., 2007). Gao et al reported that both A and B trisaccharides were located near the $\mathrm{P}$ dimer interface, suggesting that dimerization was both important for structural stability, and essential for its receptor binding functionality (Gao et al., 2016). It remains unknown if HBGAs could bind to P protein monomers. In our study, the bacterial-surface-displayed $\mathrm{P}$ protein had the ability to interact with HBGA-like molecules from romaine lettuce. It remains unknown if SD-GII.4P remains monomers or forms dimers on the surface of transformed bacteria to bind HBGA-like molecules in romaine lettuce. Currently, we are in the process of investigating whether the $\mathrm{P}$ monomers or dimers on the bacterial surface participate in the binding of HBGAs.

In this study, we showed that INP-P (GII.4) BL21 could bind to HBGA-like molecules in leaves and veins of romaine lettuce. The nature of the HBGA-like ligand in romaine lettuce remains unknown. Currently, we are in the process of developing protocols to separate the SD-GII.4P-ligand complex in order to release the ligands for characterization.

\section{CONCLUSION}

The interaction between GII.4 HuNoV and ligand candidates in romaine lettuce was confirmed by using a novel bacterial expression system that expressed P protein of GII.4 HuNoV on the surface of transformed bacteria. This new yet simple surface display system could be used in the future for the purpose of isolating and characterizing receptor/ligand candidates for HuNoVs. 


\section{AUTHOR CONTRIBUTIONS}

LQ, WD, and RS designed the experiments. WM carried out the experiments with assistance from RS, ZY, GS, and WD. RS, $\mathrm{WM}, \mathrm{TP}$, and LQ conducted statistical analysis. WM, TP, and LQ wrote the paper. WD modified the paper. All authors reviewed the results, made substantial contributions and approved the final version of the manuscript.

\section{REFERENCES}

Bozkurt, H., D’Souza, D. H., and Davidson, P. M. (2013). Determination of the thermal inactivation kinetics of the human norovirus surrogates, murine norovirus and feline calicivirus. J. Food Prot. 76, 79-84. doi: 10.4315/0362028X.JFP-12-327

Cao, S., Lou, Z., Tan, M., Chen, Y., Liu, Y., Zhang, Z., et al. (2007). Structural basis for the recognition of blood group trisaccharides by norovirus. J. Virol. 81, 5949-5957. doi: 10.1128/JVI.00219-07

Cochet, N., and Widehem, P. (2000). Ice crystallization by Pseudomonas syringae. Appl. Microbiol. Biotechnol. 54, 153-161. doi: 10.1007/s002530000377

Dicaprio, E., Ma, Y., Purgianto, A., Hughes, J., and Li, J. (2012). Internalization and dissemination of human norovirus and animal caliciviruses in hydroponically grown romaine lettuce. Appl. Environ. Microbiol. 78, 6143-6152. doi: 10.1128/ AEM.01081-12

Dicaprio, E., Purgianto, A., Ma, Y., Hughes, J., Dai, X., and Li, J. (2015). Attachment and localization of human norovirus and animal caliciviruses in fresh produce. Int. J. Food Microbiol. 211, 101-108. doi: 10.1016/j.ijfoodmicro.2015.07.013

Esseili, M. A., Wang, Q., and Saif, L. J. (2012). Binding of human GII.4 norovirus virus-like particles to carbohydrates of romaine lettuce leaf cell wall materials. Appl. Environ. Microbiol. 78, 786-794. doi: 10.1128/AEM.07081-11

Ethelberg, S., Lisby, M., Bottiger, B., Schultz, A. C., Villif, A., Jensen, T., et al. (2010). Outbreaks of gastroenteritis linked to lettuce, Denmark, January 2010. Euro Surveill. 15:19484.

Ettayebi, K., Crawford, S. E., Murakami, K., Broughman, J. R., Karandikar, U., Tenge, V. R., et al. (2016). Replication of human noroviruses in stem cellderived human enteroids. Science 353, 1387-1393. doi: 10.1126/science.aaf5211

Gandhi, K. M., Mandrell, R. E., and Tian, P. (2010). Binding of virus-like particles of Norwalk virus to romaine lettuce veins. Appl. Environ. Microbiol. 76, 7997-8003. doi: 10.1128/AEM.01566-10

Gao, X., Esseili, M. A., Lu, Z., Saif, L. J., and Wang, Q. (2016). Recognizing HBGA-like carbohydrates in lettuce by human GII.4 norovirus. Appl. Environ. Microbiol. 82, 2966-2974. doi: 10.1128/AEM.04096-15

Golberg, D., Kroupitski, Y., Belausov, E., Pinto, R., and Sela, S. (2011). Salmonella Typhimurium internalization is variable in leafy vegetables and fresh herbs. Int. J. Food Microbiol. 145, 250-257. doi: 10.1016/j.ijfoodmicro.2010.12.031

Grove, S. F., Suriyanarayanan, A., Puli, B., Zhao, H., Li, M., Li, D., et al. (2015). Norovirus cross-contamination during preparation of fresh produce. Int. J. Food Microbiol. 198, 43-49. doi: 10.1016/j.ijfoodmicro.2014.12.023

Hoa Tran, T. N., Trainor, E., Nakagomi, T., Cunliffe, N. A., and Nakagomi, O. (2013). Molecular epidemiology of noroviruses associated with acute sporadic gastroenteritis in children: global distribution of genogroups, genotypes and GII.4 variants. J. Clin. Virol. 56, 185-193. doi: 10.1016/j.jcv.2012.11.011

Jiang, X., Wang, M., Graham, D. Y., and Estes, M. K. (1992). Expression, selfassembly, and antigenicity of the Norwalk virus capsid protein. J. Virol. 66, 6527-6532.

Jones, M. K., Grau, K. R., Costantini, V., Kolawole, A. O., de Graaf, M., Freiden, P., et al. (2015). Human norovirus culture in B cells. Nat. Protoc. 10, 1939-1947. doi: 10.1038/nprot.2015.121

Kroupitski, Y., Pinto, R., Belausov, E., and Sela, S. (2011). Distribution of Salmonella typhimurium in romaine lettuce leaves. Food Microbiol. 28, 990-997. doi: 10.1016/j.fm.2011.01.007

Li, Q., Yan, Q., Chen, J., He, Y., Wang, J., Zhang, H., et al. (2012). Molecular characterization of an ice nucleation protein variant (inaQ) from Pseudomonas syringae and the analysis of its transmembrane transport activity in Escherichia coli. Int. J. Biol. Sci. 8, 1097-1108. doi: 10.7150/ijbs. 4524

\section{ACKNOWLEDGMENTS}

This work was supported by the grant No. 31301475 from the National Natural Science Foundation of China and USDA Agricultural Research Service CRIS project 5325-42000-046-06R. We would like to thank Lin $\mathrm{Li}$ for inaQn gene and David Yang for his help in editing the manuscript.

Li, Q., Yu, Z., Shao, X., He, J., and Li, L. (2009). Improved phosphate biosorption by bacterial surface display of phosphate-binding protein utilizing ice nucleation protein. FEMS Microbiol. Lett. 299, 44-52. doi: 10.1111/j.1574-6968.2009. 01724.x

Made, D., Trubner, K., Neubert, E., Hohne, M., and Johne, R. (2013). Detection and typing of norovirus from frozen strawberries involved in a large-scale gastroenteritis outbreak in Germany. Food Environ. Virol. 5, 162-168. doi: 10.1007/s12560-013-9118-0

Mok, H. F., Barker, S. F., and Hamilton, A. J. (2014). A probabilistic quantitative microbial risk assessment model of norovirus disease burden from wastewater irrigation of vegetables in Shepparton, Australia. Water Res. 54, 347-362. doi: 10.1016/j.watres.2014.01.060

Niu, M., Yu, Q., Tian, P., Gao, Z., Wang, D., and Shi, X. (2015). Engineering bacterial surface displayed Human norovirus capsid proteins: a novel system to explore interaction between norovirus and ligands. Front. Microbiol. 6:1448. doi: $10.3389 /$ fmicb.2015.01448

Sarvikivi, E., Roivainen, M., Maunula, L., Niskanen, T., Korhonen, T., Lappalainen, M., et al. (2012). Multiple norovirus outbreaks linked to imported frozen raspberries. Epidemiol. Infect. 140, 260-267. doi: 10.1017/ S0950268811000379

Tamminen, K., Huhti, L., Koho, T., Lappalainen, S., Hytönen, V. P., Vesikari, T., et al. (2012). A comparison of immunogenicity of norovirus GII-4 virus-like particles and P-particles. Immunology 135, 89-99. doi: 10.1111/j.1365-2567. 2011.03516.x

Tan, M., Fang, P., Chachiyo, T., Xia, M., Huang, P., Fang, Z., et al. (2008). Noroviral $\mathrm{P}$ particle: structure, function and applications in virus-host interaction. Virology 382, 115-123. doi: 10.1016/j.virol.2008.08.047

Tan, M., Fang, P., Xia, M., Chachiyo, T., Jiang, W., and Jiang, X. (2011). Terminal modifications of norovirus $\mathrm{P}$ domain resulted in a new type of subviral particles, the small P particles. Virology 410, 345-352. doi: 10.1016/j.virol.2010.11.017

Tan, M., and Jiang, X. (2005). The p domain of norovirus capsid protein forms a subviral particle that binds to histo-blood group antigen receptors. J. Virol. 79, 14017-14030. doi: 10.1128/JVI.79.22.14017-14030.2005

Tian, P., Brandl, M., and Mandrell, R. (2005). Porcine gastric mucin binds to recombinant norovirus particles and competitively inhibits their binding to histo-blood group antigens and Caco-2 cells. Lett. Appl. Microbiol. 41, 315-320. doi: 10.1111/j.1472-765X.2005.01775.x

Tian, P., Jiang, X., Zhong, W., Jensen, H. M., Brandl, M., Bates, A. H., et al. (2007). Binding of recombinant norovirus like particle to histo-blood group antigen on cells in the lumen of pig duodenum. Res. Vet. Sci. 83, 410-418. doi: 10.1016/j.rvsc.2007.01.017

Vega, E., Smith, J., Garland, J., Matos, A., and Pillaii, S. D. (2005). Variability of virus attachment patterns to butterhead lettuce. J. Food Prot. 68, 2112-2117.

Verhaelen, K., Bouwknegt, M., Lodder-Verschoor, F., Rutjes, S. A., and de Roda Husman, A. M. (2012). Persistence of human norovirus GII.4 and GI.4, murine norovirus, and human adenovirus on soft berries as compared with PBS at commonly applied storage conditions. Int. J. Food Microbiol. 160, 137-144. doi: $10.1016 /$ j.ijfoodmicro.2012.10.008

Vinjé, J. (2015). Advances in laboratory methods for detection and typing of norovirus. J. Clin. Microbiol. 53, 373-381. doi: 10.1128/JCM.01535-14

Wang, D., Xu, S., Yang, D., Young, G. M., and Tian, P. (2014). New in situ capture quantitative (real-time) reverse transcription-PCR method as an alternative approach for determining inactivation of Tulane virus. Appl. Environ. Microbiol. 80, 2120-2124. doi: 10.1128/AEM.04036-13

Wang, Q., Zhang, Z., and Saif, L. J. (2012). Stability of and attachment to lettuce by a culturable porcine sapovirus surrogate for human caliciviruses. Appl. Environ. Microbiol. 78, 3932-3940. doi: 10.1128/AEM.06600-11 
Wei, J., Jin, Y., Sims, T., and Kniel, K. E. (2010). Manure- and biosolidsresident murine norovirus 1 attachment to and internalization by Romaine lettuce. Appl. Environ. Microbiol. 76, 578-583. doi: 10.1128/AEM. 02088-09

Xicohtencatl-Cortes, J., Sanchez Chacon, E., Saldana, Z., Freer, E., and Giron, J. A. (2009). Interaction of Escherichia coli O157:H7 with leafy green produce. J. Food Prot. 72, 1531-1537.

Xu, S., Wang, D., Yang, D., Liu, H., and Tian, P. (2015). Alternative methods to determine infectivity of Tulane virus: a surrogate for human nororvirus. Food Microbiol. 48, 22-27. doi: 10.1016/j.fm.2014. 12.004
Conflict of Interest Statement: The authors declare that the research was conducted in the absence of any commercial or financial relationships that could be construed as a potential conflict of interest.

Copyright $\odot 2017$ Wang, Rong, Tian, Zhou, Guan, Li and Wang. This is an openaccess article distributed under the terms of the Creative Commons Attribution License (CC BY). The use, distribution or reproduction in other forums is permitted, provided the original author(s) or licensor are credited and that the original publication in this journal is cited, in accordance with accepted academic practice. No use, distribution or reproduction is permitted which does not comply with these terms. 